\title{
Changes in Gene Expression and Metabolite Profiles in Platanus acerifolia Leaves in Response to Feeding Damage Caused by Corythucha ciliata
}

\author{
Fengqi $\mathrm{Li}^{1{ }^{1+}}{ }^{+}$Chunyan $\mathrm{Wu}^{2,+}$, Youssef Dewer ${ }^{3}, \mathrm{Du} \mathrm{Li}^{1}$, Cheng $\mathrm{Qu}{ }^{1}$ and Chen Luo ${ }^{1, *}$ \\ 1 Institute of Plant and Environment Protection, Beijing Academy of Agriculture and Forestry Sciences, \\ Beijing 100097, China \\ 2 College of Life Sciences, Capital Normal University, Beijing 100037, China \\ 3 Bioassay Research Department, Central Agricultural Pesticide Laboratory, Sabahia Plant Protection Research \\ Station, Agricultural Research Center, Alexandria 21616, Egypt \\ * Correspondence: luochen1010@126.com \\ + These authors contributed equally to this work.
}

Received: 24 April 2019; Accepted: 28 June 2019; Published: 15 July 2019

\begin{abstract}
The sycamore lace bug, Corythucha ciliata (Say) is a highly invasive pest insect that feeds on sycamore trees (Platanus spp.) worldwide. The interaction between Platanus species and this insect pest has not yet been studied at the molecular level. Therefore, a recent study was conducted to compare the gene expression and metabolite profiles of Platanus acerifolia leaves in response to C. ciliata feeding damage after 24 and $48 \mathrm{~h}$. We employed high throughput RNA sequencing (RNA- seq) to identify a total of 2,828 significantly differentially expressed genes (DEGs) after C. ciliata feeding. In addition, 303 unigenes were found to be up-regulated at both time points. Moreover, Kyoto Encyclopedia of Genes and Genomes (KEGG) pathway enrichment analysis showed that monoterpenoid biosynthesis, the linoleic acid metabolism pathway, and alpha- linolenic acid metabolism were the most prominent pathways among the DEGs. Further analysis of the metabolite profiles showed that nine metabolites were significantly different before and after $C$. ciliata damage. In addition, we analyzed DEGs detected in the P. acerifolia and C. ciliata interaction using Mapman. The terpene synthase gene family was also identified. We suggest that the results obtained from DEGs and metabolite analysis can provide important information for the identification of genes involved in the P. acerifolia-C. ciliata interaction, which might be necessary for controlling C. ciliata efficiently.
\end{abstract}

Keywords: Platanus acerifolia; Corythucha ciliata; RNA-seq; differentially expressed genes (DEGs); metabolites

\section{Introduction}

Platanus L. is an important genus of large trees commonly known as sycamore or plane trees. Platanus species are widely planted all over China as shade trees due to their large spreading crown, and also for their rapid growth and pruning resistance. The sycamore lace bug, Corythucha ciliata (Say, 1832) (Hemiptera: Tingidae), which is native to the central and eastern parts of North America, was subsequently introduced into Europe and Asia [1,2]. This pest has a strong ability to spread and is considered to be a new alien invasive insect pest in China [2]. C. ciliata specifically damages members of the Platanus genus, including Platanus occidentalis, P. acerifolia, and P. orientalis. The most important relationship between $P$. acerifolia and $C$. ciliata is that $P$. acerifolia is the most widely planted Platanus species in China [3]. C. ciliata was first discovered in Changshan, China in 2002. So far, C. ciliata has been observed to cause damage to P. acerifolia trees in Hunan, Hubei, Shanghai, Zhejiang, Jiangsu, Shandong, Henan, Chongqing, Guizhou, and Beijing in China [2,4], and it has been included in the list of forest pests in China. C. ciliata infestation can cause growth disruption, weakness, and even death in 
P. acerifolia, and it has seriously affected landscaping in many Chinese cities. Currently, the management of $C$. ciliata still mainly depends on chemical insecticides [5]. However, the P. acerifolia is a widely planted street tree in urban areas, so the use of pesticides does not only cause serious environmental pollution but could harm human health. Therefore, to understand the interaction mechanism between $P$. acerifolia and C. ciliata would provide a great help to accelerate the development of an effective environmentally friendly pest management strategy to manipulate the populations of $C$. ciliata which is also problematic for other Asian and European countries.

Unlike animals, higher plants, which are sessile, cannot escape from the surroundings, and adapt themselves to the changing environments by a series of molecular responses aimed to cope with these challenges. The physiological basis for these molecular responses is the integration of many transduced events into a comprehensive network of signaling pathways. Consequently, plants have developed various defense strategies to respond to insects feeding on them [6,7]. These include changes in hormone levels, production of terpene volatiles that can affect insect behavior, and the production of flavonoid secondary metabolites [8,9]. In this way, plants enhance their resistance to pests. In a word, it is at the molecular level-gene expression and the control in time and space that we can bring out the mystery of living organisms and explore the nature of environmental changes.

In order to study the molecular biology of the P. acerifolia and C. ciliata interaction, C. ciliata were allowed to feed individually on the leaves of P. acerifolia, and then we combined RNA-seq and metabolite analysis to study the response of P. acerifolia to $C$. ciliata damage at the gene expression level. Differences in P. acerifolia leaf metabolite profiles were detected using metabolomic techniques. Finally, the key genes responsible for defense against this pest, the possible signaling pathways, and defensive compounds were identified. The results of our study provide basic knowledge for designing novel strategies to control C. ciliata in the future.

\section{Results}

\subsection{Transcriptome Sequencing and RNA-seq Read Assembly}

The newly emerged C. ciliata adults were allowed to feed individually on P. acerifolia leaves for 24 and $48 \mathrm{~h}$. RNA was then extracted from the leaf tissue samples (three biological replicates at each of two different time points after infestation of $C$. ciliata to $P$. acerifolia leaves). The control group was represented by leaves not infested by insects. Also, 12 cDNA libraries were constructed for RNA sequencing. The sequencing data is stored in the NCBI Sequence Read Archive (SRA, http://www.ncbi.nlm.nih.gov/Traces/sra); under BioProject ID number PRJNA484863 and accession numbers SRR8631807 to SRR8631818. High throughput DNA sequencing generated $7.08 \times 10^{9}$ to $8.66 \times 10^{9}$ raw bases per sample, of which $>93 \%$ had Q-scores between 20 and 30 (Table 1). The total length of the combined reads from the 12 samples was $2.02 \times 10^{8} \mathrm{bp}$, and there were 199,080 unigenes with an average length of $1015.15 \mathrm{bp}$ and N50 of $1579 \mathrm{bp}$ (Table 2). The Mean reads mapped to the trinity reference assemble were $1442.764 \mathrm{bp}$.

Table 1. Output RNA-seq statistics from leaf samples of P. acerifolia.

\begin{tabular}{ccccc}
\hline Sample & Raw Bases & Clean Bases & Q20 (\%) & Q30 (\%) \\
\hline CK24h_1 & $8.66 \times 10^{9}$ & $8.46 \times 10^{9}$ & 97.91 & 93.70 \\
\hline CK24h_2 & $8.24 \times 10^{9}$ & $8.06 \times 10^{9}$ & 98.10 & 94.13 \\
\hline CK24h_3 & $7.51 \times 10^{9}$ & $7.35 \times 10^{9}$ & 97.76 & 93.31 \\
\hline CK48h_1 & $8.18 \times 10^{9}$ & $8.00 \times 10^{9}$ & 97.68 & 93.17 \\
\hline CK48h_2 & $7.08 \times 10^{9}$ & $6.94 \times 10^{9}$ & 98.22 & 94.41 \\
\hline CK48h_3 & $8.45 \times 10^{9}$ & $8.25 \times 10^{9}$ & 97.89 & 93.64 \\
\hline T24h_1 & $8.00 \times 10^{9}$ & $7.81 \times 10^{9}$ & 97.71 & 93.22 \\
\hline
\end{tabular}


Table 1. Cont.

\begin{tabular}{ccccc}
\hline Sample & Raw Bases & Clean Bases & Q20 (\%) & Q30 (\%) \\
\hline T24h_2 & $7.55 \times 10^{9}$ & $7.40 \times 10^{9}$ & 98.13 & 94.20 \\
\hline T24h_3 & $8.15 \times 10^{9}$ & $7.97 \times 10^{9}$ & 97.99 & 93.87 \\
\hline T48h_1 & $8.41 \times 10^{9}$ & $8.23 \times 10^{9}$ & 97.92 & 93.70 \\
\hline T48h_2 & $8.09 \times 10^{9}$ & $7.90 \times 10^{9}$ & 97.84 & 93.52 \\
\hline T48h_3 & $7.92 \times 10^{9}$ & $7.75 \times 10^{9}$ & 98.14 & 94.21 \\
\hline
\end{tabular}

Table 2. Statistics for the transcriptome assemblies derived from P. acerifolia leaf RNA.

\begin{tabular}{ccccccccc}
\hline Type & $\begin{array}{c}\text { Transcripts } \\
\text { num }\end{array}$ & $\begin{array}{c}\text { Unigenes } \\
\text { num }\end{array}$ & $\begin{array}{c}\text { Total } \\
\text { Sequence } \\
\text { Base }\end{array}$ & Largest & Smallest & $\begin{array}{c}\text { Average } \\
\text { Length }\end{array}$ & $\begin{array}{c}\text { Mean } \\
\text { N50 } \\
\text { Mapped } \\
\text { Reads }\end{array}$ \\
\hline Resource & 199080 & 121136 & $2.02 \times 10^{8}$ & 18931 & 201 & 1015.15 & 1579 & 1442.764 \\
\hline
\end{tabular}

\subsection{Identification of DEGs in Response to C. ciliata Feeding Damage}

To analyze the gene expression patterns induced by C. ciliata feeding in the 24 and $48 \mathrm{~h}$ periods, we further examined the differential gene expression by comparing gene expression values (Transcripts Per Million, TPM) between the feeding treatments vs. the controls at each time point. A p-adjust value $\leq 0.05$ and $\mid \log 2$ fold change $\mid \geq 1$ were set as the thresholds to detect significant DEGs. A total of 2790 DEGs was identified between the $24 \mathrm{~h}$ treatment and the control (Figure 1). Among these, 1907 unigenes were up-regulated, and 883 unigenes were down-regulated. Furthermore, 1809 unigenes (921 up-regulated and 888 down-regulated) were expressed differentially after the $48 \mathrm{~h}$ treatment (Figure 1). In addition, we analyzed the DEGs that overlapped between these two treatment time points. The results revealed that there were 303 and 35 up- and down-regulated DEGs that overlapped in the $24 \mathrm{~h}$ and $48 \mathrm{~h}$ treatments, respectively (Figure 1). Also, 2326 and 1345 DEGs were distinctive in the $24 \mathrm{~h}$ and $48 \mathrm{~h}$ treatments, respectively (Figure 1 ).

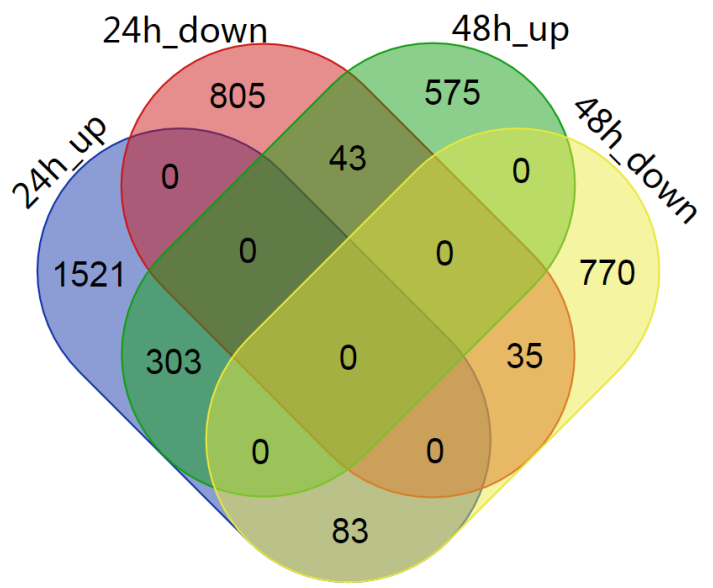

Figure 1. Venn diagram showing the DEGs in P. acerifolia leaves in response to C. ciliata damage after 24 and $48 \mathrm{~h}$ of feeding.

\subsection{GO Enrichment Analysis of the DEGs}

To investigate the functions of the DEGs detected in the P. acerifolia response to C. ciliata feeding, we analyzed the DEGs by Gene Ontology (GO) term enrichment. For the $24 \mathrm{~h}$ treatment, the significantly enriched GO terms in the DEGs, 159 GO terms were significantly enriched, the ten most significant terms were: oxidoreductase activity, acting on single donors with incorporation of molecular oxygen, incorporation of two atoms of oxygen (GO:0016702), dioxygenase activity 
(GO:0051213), monocarboxylic acid biosynthetic process (GO:0072330), lipid biosynthetic process (GO:0008610), terpene synthase activity (GO:0010333), fatty acid metabolic process (GO:0006631), organic acid biosynthetic process (GO:0016053), carboxylic acid biosynthetic process (GO:0046394), monooxygenase activity (GO:0004497), heme binding (GO:0020037) (Table S1 and Figure S1). In the $48 \mathrm{~h}$ treatment, $187 \mathrm{GO}$ terms were significantly enriched, the 10 most significant terms were: protein-chromophore linkage (GO:0018298), cell wall (GO:0005618), external encapsulating structure (GO:0030312), glucosamine-containing compound catabolic process (GO:1901072), glucosaminecontaining compound metabolic process (GO:1901071), chitin metabolic process (GO:0006030), amino sugar catabolic process (GO:0046348), chitin catabolic process (GO:0006032), aminoglycan metabolic process (GO:0006022), aminoglycan catabolic process (GO:0006026) (Table S2 and Figure S2). Also, for the 303 unigenes that were up-regulated at both time points, 86 GO terms were significantly enriched, the ten most significant terms were: carbon-oxygen lyase activity, acting on phosphates (GO:0016838), monocarboxylic acid biosynthetic process (GO:0072330), magnesium ion binding (GO:0000287), oxylipin biosynthetic process (GO:0031408), oxylipin metabolic process (GO:0031407), monooxygenase activity (GO:0004497), oxidoreductase activity, acting on paired donors, with incorporation or reduction of molecular oxygen (GO:0016705), single-organism catabolic process (GO:0044712), oxidoreductase activity, acting on single donors with incorporation of molecular oxygen (GO:0016701), dioxygenase activity (GO:0051213) (Table S3 and Figure 2).

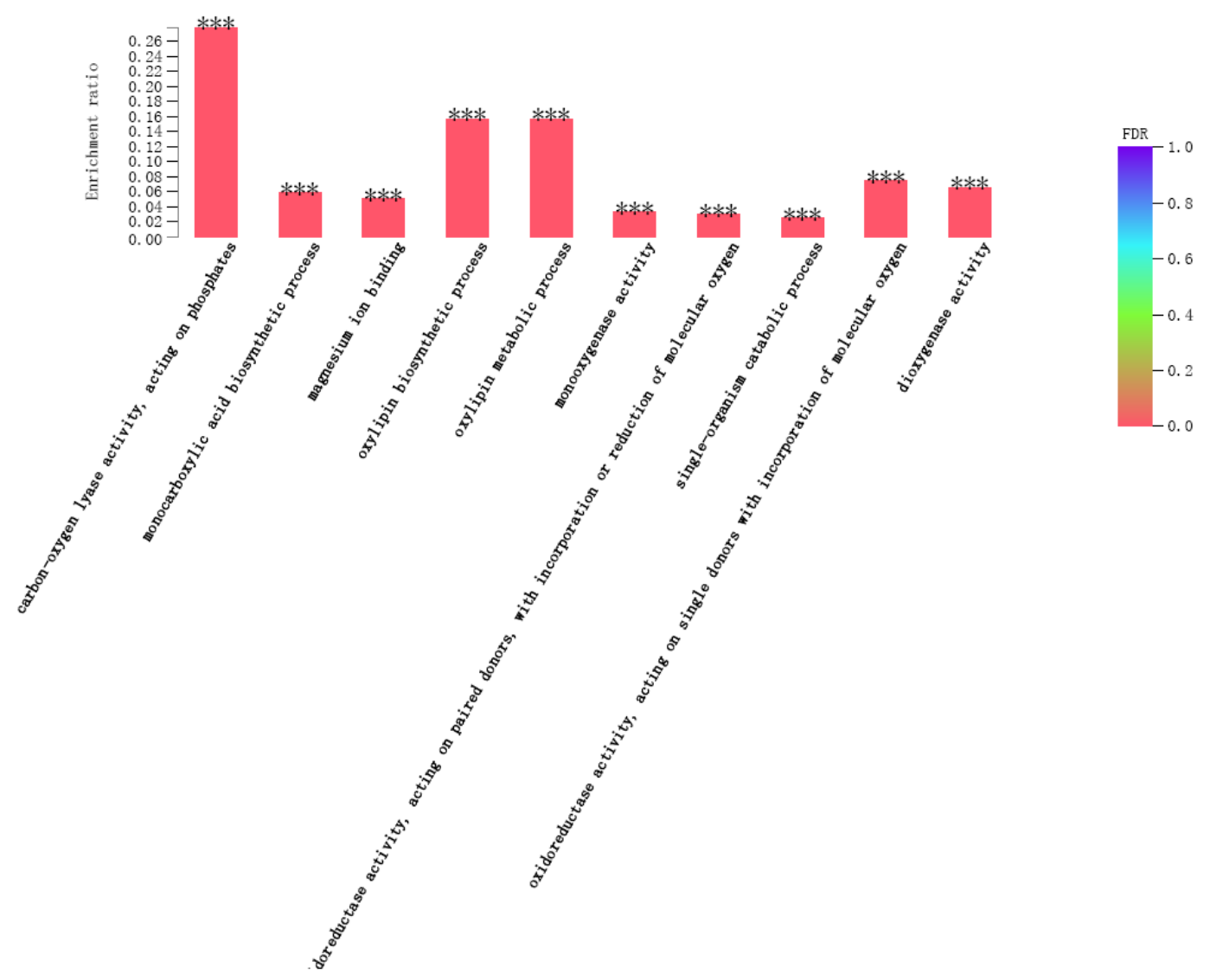

Figure 2. Top ten GO terms that were enriched in the 303 unigenes that were up-regulated at both time points. The $\mathrm{x}$-axis represents the GO term and the $\mathrm{y}$-axis represents the enrichment ratio (the number of unigenes annotated to a GO term in a gene set divided by the number of unigenes annotated to this GO term in the entire unigenes as background set.) The color indicates the significance of the enrichment. *** mean False Discovery Rate $<0.001$. 


\subsection{KEGG Enrichment Analysis of the DEGs}

We used KEGG pathway analysis to map the DEGs to the biochemical pathways involved in the plant response to C. ciliata damage. "Monoterpenoid biosynthesis", "Linoleic acid metabolism" and "alpha-Linolenic acid metabolism" were prominent pathways between the treatments and controls in response to C. ciliata feeding damage after 24 and $48 \mathrm{~h}$ (Tables $S 4$ and S5). In addition to these three pathways, "Phenylpropanoid biosynthesis", "Phenylalanine, tyrosine and tryptophan biosynthesis", "Flavonoid biosynthesis", "Terpenoid backbone biosynthesis", and "Sesquiterpenoid and triterpenoid biosynthesis" are five of 26 pathways that were enriched at $24 \mathrm{~h}$ (Table S4). In the $48 \mathrm{~h}$ treatment, 22 pathways were enriched, including "Photosynthesis-antenna proteins", "Photosynthesis", "MAPK signaling pathway-plant", "Cutin, suberine and wax biosynthesis", and "Pentose and glucuronate interconversions" (Table S5). For the 303 unigenes that were up-regulated at both timepoints, there were 17 enriched pathways. Of these 17 pathways, the first three prominent pathways of significance are "Monoterpenoid biosynthesis", "Linoleic acid metabolism pathway" and "alpha-Linolenic acid metabolism" (Table S6). The results of the above analyses indicate that these pathways may be involved in the interaction between P. acerifolia and C. ciliata.

\subsection{MapMan Overview of DEGs Related to Plant-Pest Interaction}

To reveal the role of DEGs in the plant response to C. ciliata feeding damage, we used the MapMan package to investigate the P. acerifolia biotic stress pathways. An overview of the role played by biotic stress in the regulation of DEGs, such as hormone signaling genes, Transcription Factors (TFs), Mitogen-Activated Protein Kinases (MAPK), Pathogenesis-Related (PRs), and PR-proteins, is shown in Figure 3. After $24 \mathrm{~h}$ of feeding, most of the DEGs belonging to JA pathways were up-regulated, as were most of the WRKY, myeloblastosis (MYB), and Ethylene Responsive Factor (ERF)-related DEGs, while all DEGs involved in SA signaling were down-regulated (Figure 3A). After $48 \mathrm{~h}$, one ABA-related DEG and most of the DEGs belonging to redox state, peroxidase, and glutathione-S-transferase pathways were up-regulated, while one most of the DGEs belonging to known TF families such as WRKY, ERF, and DNA-binding with one finger (DOF) and two of the three heat shock proteins genes were down-regulated (Figure 3B). A detailed list of all the DEGs involved in the P. acerifolia-C. ciliata interaction and their MapMan functional categories is given in Table S7.

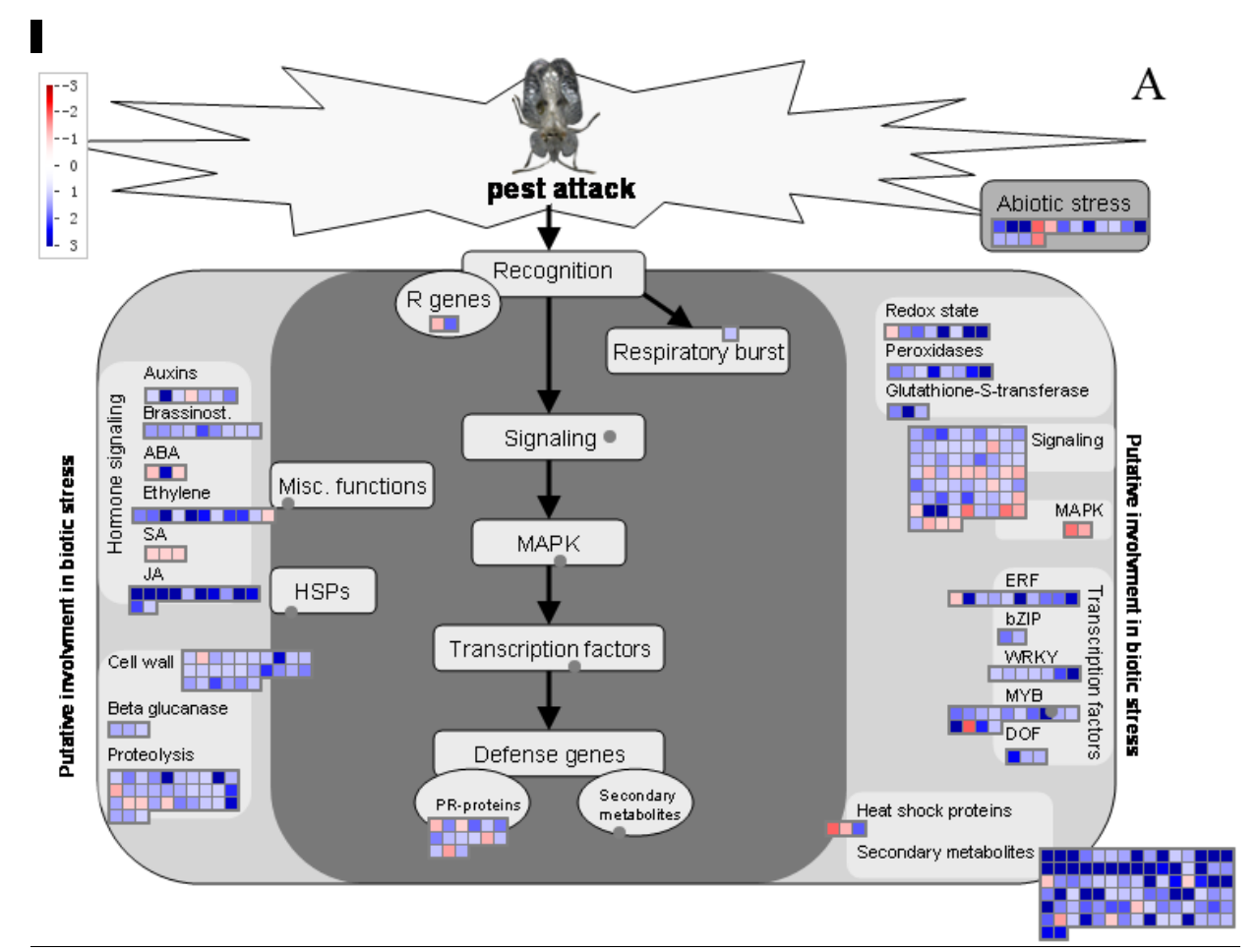

Figure 3. Cont. 


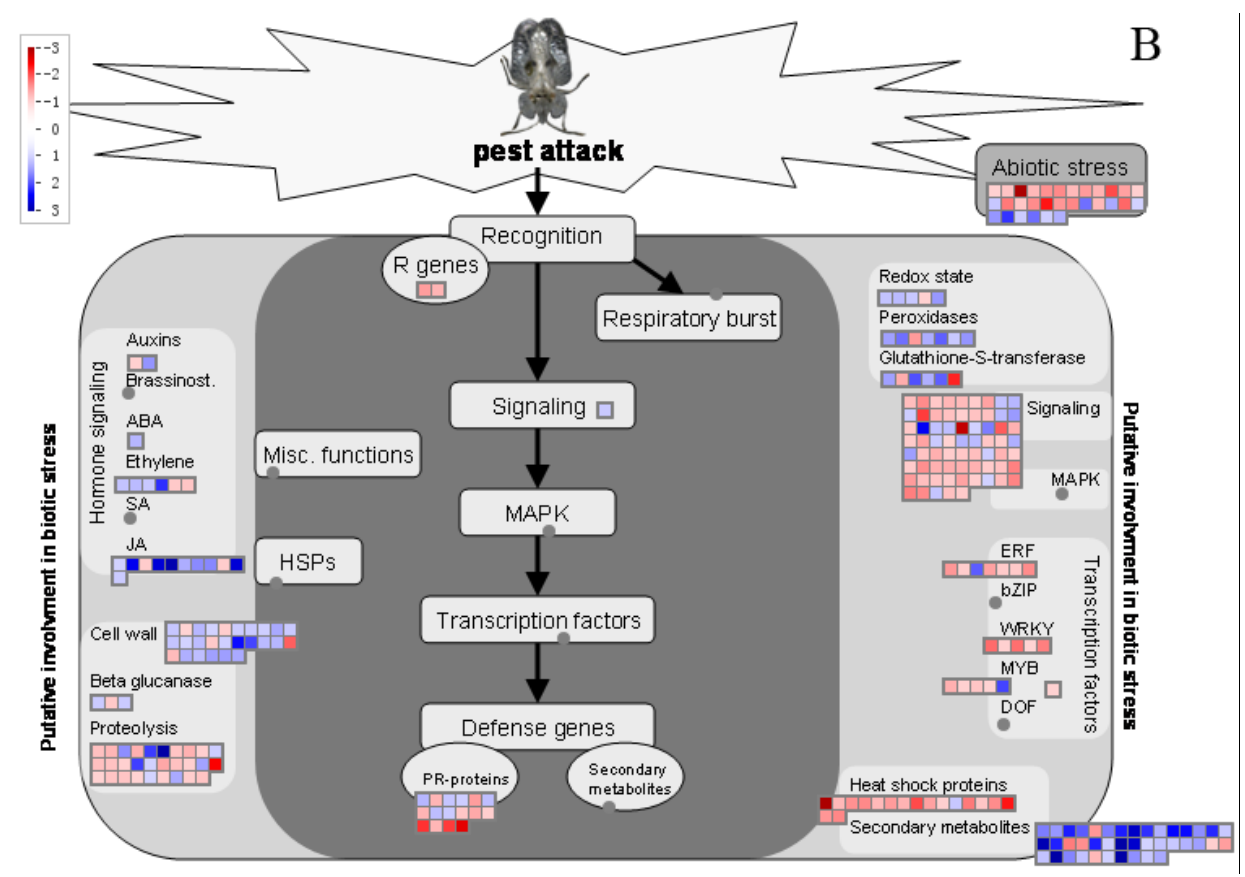

Figure 3. MapMan visualization of DEGs in the P. acerifolia response to C. ciliata feeding damage. The up- and down-regulated genes are indicated in red and blue colors, respectively. The $\log _{2} \mathrm{FC}$ (treatment TPM/mock TPM) are shown in the scale bars. (A) 24-h treatment. (B) 48-h treatment.

\subsection{Response of Terpene Synthase Gene Expression to C. ciliata Damage}

Terpene synthase genes catalyze the biosynthesis of terpenoid volatiles in P. acerifolia leaves. We identified 14 putative terpene synthase (TPS) genes from our RNA-seq dataset. Among them, seven showed significantly up-regulated expression after both 24 and $48 \mathrm{~h}$ of $C$. ciliata feeding. These upregulated TPS genes may be involved in enhancing resistance to $C$. ciliata by producing volatiles that either repel this pest or attract its natural enemies. To examine the phylogenetic relationships among these TPS proteins and those from several other species, we constructed an unrooted neighbor-joining phylogenetic tree. The TPS genes were classified into five different families (clades) named TPS-b to TPS-g (Figure S3 and Table S8).

\subsection{Metabolite Changes in P. Acerifolia Leaves in Response to C. ciliata Feeding Damage}

To analyze the changes in metabolite profiles that occur in response to C. ciliata feeding, we compared the metabolic compounds isolated from P. acerifolia leaves after C. ciliata adults were allowed to feed for 24 and $48 \mathrm{~h}$. We detected 50 metabolites in these samples and performed Principal component analysis (PCA) (Figure S4) and Partial least squares discriminant analysis (PLSDA) (Figure 3A) analyses. As shown in Figure S4 and Figure 4A, the C. ciliata feeding treatments and the controls can be distinguished by their metabolite profiles. After ANOVA analysis, we found that the levels of nine metabolites were significantly different $(p<0.05)$; these included glycoside, cinnamate, indole- 3- acetamide, L-tryptophan, epigallocatechin, L-fucitol and three amino acids (L-lysine, L- valine, and serine) (Figure 4B). Flavonoids are often closely related to insect interactions in plants. In this study, we detected one flavonol chemical, epigallocatechin, which is increased at $24 \mathrm{~h}$ and decreased at $48 \mathrm{~h}$. Combining the RNA-seq datasets, we detected seven key genes for epigallocatechin biosynthesis, including three genes involved in the biosynthesis of delphinidin from leucodelphinidin, and four genes that catalyze the biosynthesis of epigallocatechin from delphindin (Figure 5). In addition, the relative expression values of these genes are consistent with the observed changes in epigallocatechin levels (Figure 5). These genes should be candidates for the key genes in the epigallocatechin biosynthesis pathway. 


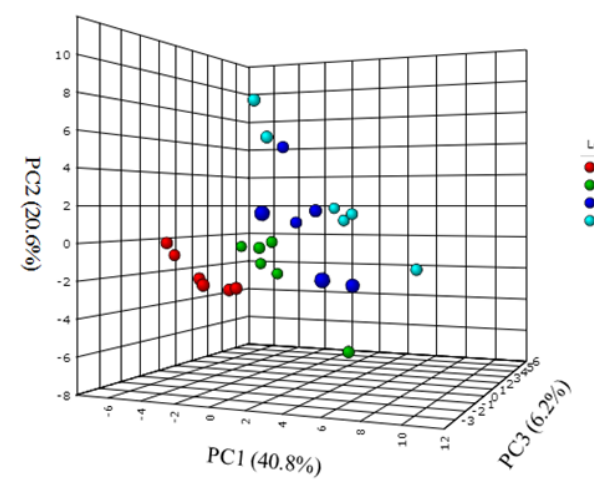

B

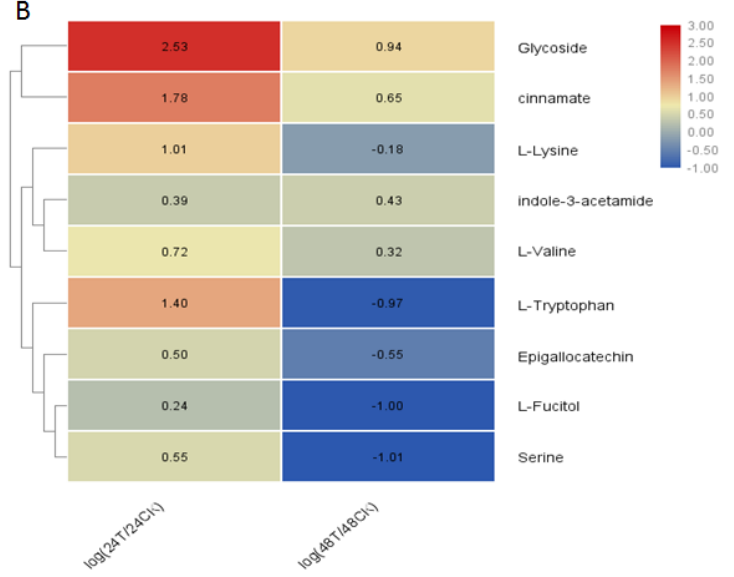

Figure 4. PLSDA and the changes in metabolite profiles in P. acerifolia leaves in response to C. ciliata feeding damage. (A) PLSDA of 50 metabolites. (B) Changes in nine significantly different metabolites between the 24 and $48 \mathrm{~h}$ treatments and controls. The numbers in the heat map represent the log values of the ratios between treatments and controls.<smiles>Oc1cc(O)c2c(c1)O[C@H](c1cc(O)c(O)c(O)c1)C(O)C2O</smiles><smiles>Oc1cc(O)c2cc(O)c(-c3cc(O)c(O)cc3O)cc2c1</smiles><smiles>Oc1cc(O)c2c(c1)OC(O)CC2c1cc(O)c(O)cc1O</smiles>

Leucodelphinidin

TRINITY_DN16916_c0_g1

TRINITY_DN106074_c2_g1

TRINITY_DN106074_c6_g1

Delphinidin

TRINITY_DN127670_c0_g1

TRINITY_DN109640_c1_g1

TRINITY_DN105568_c0_g1

TRINITY_DN197619_c0_g1 $\log (24 T / 24 C K) \quad \log (48 T / 48 C K)$

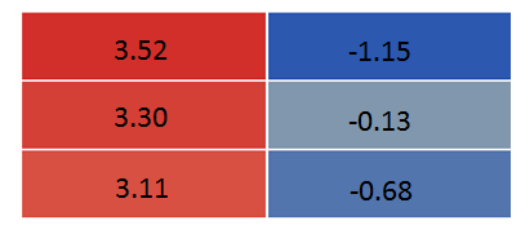

$\log (24 T / 24 C K) \quad \log (48 T / 48 C K)$

\begin{tabular}{|c|c|}
\hline 1.29 & -0.06 \\
\hline 1.68 & -0.38 \\
\hline 1.30 & -0.43 \\
\hline 1.94 & 0.10 \\
\hline
\end{tabular}

Epigallocatechin

Figure 5. Candidate genes in the epigallocatechin biosynthesis pathway. The numbers in the heat map represent the log values of the ratios between the treatments and controls.

\subsection{Validation of RNA-seq Results by qRT-PCR}

To validate the unigene TPM values obtained by RNA-seq, the expression of 32 unigenes was tested using qRT-PCR assays. The primers were listed in Table S9. These unigenes included TPS genes, and genes for heat shock proteins, peroxidases, and ethylene-responsive transcription factors. We observed a strong significant correlation between the RNA-seq TPM values and the qRT-PCR expression data (Pearson's $r$ correlation coefficient $=0.905, p<0.01$ ) (Figure 6). These results shown that the gene expression profiles determined by RNA-seq in this study are reliable. 


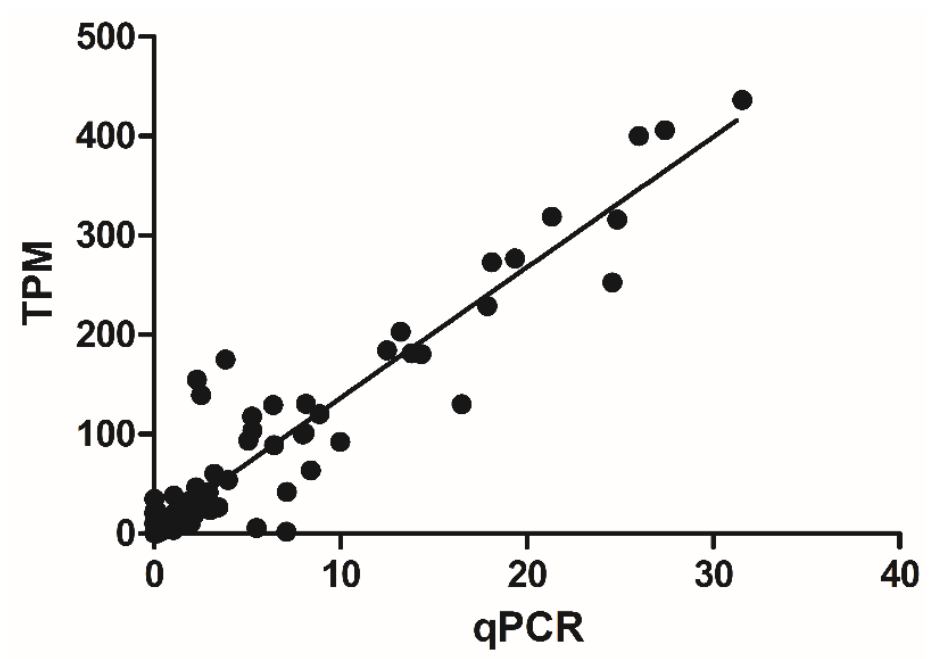

Figure 6. Correlation analysis between gene expression values obtained from RNA-seq and qRT-PCR. The value of qRT-PCR (x-axis) were plotted against RNA-seq values (y-axis).

\section{Discussion}

The sycamore lace bug, $C$. ciliata, is an important insect pest that has been causing damage to $P$. acerifolia trees in recent years [2]. C. ciliata usually feeds on the undersides of $P$. acerifolia leaves, and the leaves develop white spots at the beginning of feeding at the upper side that can cause the leaves to appear chlorotic. The damage caused by this insect pest can reduce leaf photosynthesis, leading to a decline in some physiological and biochemical indexes such as leaf photosynthetic rate, stomatal conductance, transpiration rate, chloroplast pigments and soluble sugars. C. ciliata can only complete its life cycle on the sycamore plant. Based on the importance of the host plant and the limited host specificity of this pest, studying the molecular mechanisms behind the P. acerifolia- C. ciliata interaction is important for understanding the expression of resistance in Platanus species to this insect pest.

By analyzing gene expression profiles at two different time points, we identified 1907 and 921 unigenes that were significantly up-regulated after 24 and $48 \mathrm{~h}$ of feeding, respectively. Previous studies have shown that insect stress can induce massive changes at the transcriptional level in some plants [10-12]. These changes can reprogram primary and secondary metabolic pathways, activating defense genes and signaling pathways to respond to insect attack. The large number of genes that showed changes in expression in our transcriptome are consistent with these findings. The results of our study establish a signature for $C$. ciliata-induced changes to the leaf transcriptome of $P$. acerifolia.

A total of 303 genes were up-regulated in leaves after both 24 and $48 \mathrm{~h}$ of $C$. ciliata feeding. These 303 genes are the key core genes expressed during the interaction between P. acerifolia and C. ciliata. Combining the GO, KEGG enrichment analysis, and MapMan results, we speculated that JA-related pathways and terpenoids play an important role in this plant-insect interaction system. The plant hormone JA plays vital roles in regulating the plant response to various insect stresses [13]. In our experiments, JA-related genes were induced in response to $C$. ciliata feeding damage. This indicates that in P. acerifolia, JA signal transduction may act through a mechanism similar to that in other plants. At the same time, in addition to JA pathway-related genes, we also identified related functional genes, especially genes that encode peroxidases and a series of transcription factors. The identification of these genes will provide the basis for the subsequent development of insect-resistant Platanus trees through genetic screening and possibly through genetic transformation.

The controlled production of plant volatiles plays an important role in the indirect plant defense response against insects [13]. Following pest damage, plants will release a series of volatiles, such as terpenes, to repel the insect pests and attract natural enemies, thereby improving their insect resistance to protect themselves [7]. In our previous study, we evaluated the types and contents of volatiles in leaves of P. acerifolia damaged by C. ciliata [6]. Four chemicals, including cis-3-hexen-1-ol, 1,8-cineole, 
(E)-4,8-dimethyl-1, 3,7-nonatriene, and trans- $\beta$-caryophyllene were detected in undamaged leaves. In addition, another five chemicals, $\alpha$-thujene, sabinene, myrcene, cis-3-hexenyl acetate, and $\gamma$-terpinene, were induced in leaves after feeding by $C$. ciliata. Among these volatile chemicals, cis-3-hexen-1-ol and 1,8 -cineole were found to significantly attract $C$. ciliata, whereas trans- $\beta$-caryophyllene repelled this insect pest [14]. Our study further identified 14 genes involved in the biosynthesis of these compounds. Seven genes showed significant up-regulation at both 24 and $48 \mathrm{~h}$ after feeding. These genes are candidate synthase genes for the above terpene volatiles. However, their function needs to be confirmed by protein expression and purification, in vitro enzymatic assays using geranyl diphosphate (GPP), farnesyl diphosphate (FPP), and geranylgeranyl diphosphate (GGPP) as substrates, and GC-MS analysis.

Plant secondary metabolites also play important roles in plant-insect interactions [15]. Insects often identify these metabolites by taste to determine whether the plant can provide the corresponding nutrient resources. At the same time, plants can also negatively affect insect digestion and development by producing certain secondary metabolites, thereby achieving functional insect resistance [16-18]. Flavonoid secondary metabolites are particularly important in this type of interaction $[17,18]$. In this study, we found that the epigallocatechin content increased significantly after $24 \mathrm{~h}$ of feeding, although it had decreased by $48 \mathrm{~h}$. Previous studies on epigallocatechin have shown that this compound is involved in plant resistance to diseases [19]. Considering that the interaction between plants and phloem-feeding insects has many similarities with the resistance of plants to pathogens [20,21], we hypothesize that epigallocatechin plays an important role in the interaction between P. acerifolia and C. ciliata. We also identified the candidate key enzymes involved in the biosynthesis of this compound by analyzing our leaf transcriptome dataset. Functional studies on the proteins encoded by these candidate genes need to be performed to determine their roles in epigallocatechin biosynthesis. A biological study should be carried out to observe the effects of epigallocatechin on feeding, development, and oviposition by C. ciliata.

Our study examined the intrinsic mechanisms underlying the interaction between P. acerifolia and C. ciliata using transcriptome sequencing and metabolite analysis. The high-throughput RNA sequencing technology used in this study provides a comprehensive look at the transcriptome and does not rely on the limited genomic resources presently available for Platanus species. It is worth noting that this study was performed on detached leaves and this could not fully represent the response of leaves on the tree under natural conditions. In the future, similar studies using leaves under natural conditions are needed to further confirm the key candidate genes and compounds found in the study. Additionally, further functional research on DGEs identified here should be carried out in order to clarify their specific roles in plant-insect interactions, thereby enabling the development of insect-resistant sycamore trees and providing a basis for the development of strategies to protect sycamore trees and to manage $C$. ciliata infestations.

\section{Materials and Methods}

\subsection{Plant Materials and Insects}

Corythucha ciliata nymphs were collected from P. acerifolia trees planted at the Beijing Forestry University in Haidian District, Beijing. The nymphs were raised indoors on fresh $P$. acerifolia leaves at room temperature $\left(25^{\circ} \mathrm{C} \pm 2{ }^{\circ} \mathrm{C}\right)$, a relative humidity of $50 \%-70 \%$, and a $16 \mathrm{~h}$ day $/ 8 \mathrm{~h}$ night photoperiod. The P. acerifolia leaves used in the experiments were collected from the Institute of Plant Protection of the Chinese Academy of Agricultural Sciences in Haidian District, Beijing. Only healthy and untreated leaves were selected for the experiments. The leaves were removed from the tree by cutting the base of the petiole with a sterile scalpel, and they were inserted into narrow glass test tubes containing $10 \mathrm{~mL}$ sterile water. After the insertion of the leaf, the petioles were sealed with absorbent cotton and placed in a $2 \mathrm{~L}$ glass beaker. One-hundred newly-emerged C. ciliata adults were placed on each leaf. The experiments were carried out in artificial climate chambers (the experimental conditions were the same as the above-mentioned insect feeding conditions). The measurements in three biological replicates at each of two different time points after infestation of $C$. ciliata to P. acerifolia leaves ( 24 and 
$48 \mathrm{~h})$ were performed. The control group was represented by leaves not infested by insects. After the feeding periods were finished, the insects on the leaves were removed with a soft brush. RNA was extracted from the leaves for RNA-sequencing and qRT-PCR assays.

\subsection{RNA-Sequencing and $q R T-P C R$}

Total RNA was isolated from plant tissues (leaves) using the Trizol reagent (Ambion, Life Technologies, California, USA), and RNA-seq libraries were prepared according to the manufacturer's protocol (Illumina Inc. San Diego, CA, USA). The libraries were then sequenced on an Illumina Hiseq 2500 instrument (Illumina, San Diego, CA) to produce 150 bp paired-end reads. qRT-PCR assays were conducted according to our previously described method [22]. Primers were designed using the IDT Primer Quest web tool (http://www.idtdna.com/primerquest/Home/Index). The cDNA was synthesized by Prime Script RT Reagent Kit (Perfect Real Time) (TaKaRa Bio Inc. Dalian, China). The qRT-PCR was performed using GoTaq 2-Step qRT-PCR System (Promega, Madison, WI, USA) and on the ABI Prism1 7500 (Applied Biosystems, Carlsbad, CA, USA). qRT-PCR was performed with the follow PCRcycling condition: $95^{\circ} \mathrm{C}$ for $10 \mathrm{~s}$, followed by 40 cycles of $95^{\circ} \mathrm{C}$ for $30 \mathrm{~s}$ and $60^{\circ} \mathrm{C}$ for $1 \mathrm{~min}$. Two housekeeping genes, actin (TRINITY_DN78316_c0_g1) and Alpha tubulin (TRINITY_DN106409_c0_g1), were selected to use as reference genes for normalization of gene expression in the qRT-PCR analysis. The relative expression levels of each gene were evaluated by the comparative $2^{-\Delta \Delta C t}$.

\subsection{Bioinformatics Analysis}

We first used fastp [23] to remove the reads that contained adaptor sequences, low quality bases, and undetermined bases (Ns). The sequencing quality was then assessed using FastQC (http://www.bioinformatics.babraham.ac.uk/projects/fastqc/). All downstream analyses were based on high quality, clean sequencing read data. De novo assembly of the transcriptome was performed with Trinity 2.4.0 with default parameter [24]. All assembled unigenes were used as queries to search the non-redundant $(\mathrm{Nr})$ protein database of all included species (http://www.ncbi.nlm.nih.gov/) by blastx with a threshold of $\mathrm{E}<0.00001$. RSEM was used to determine the expression levels for unigenes by calculating TPM [25]. The differentially expressed unigenes were selected using the criteria of $\log 2$ (fold change) $>1$ or $\log 2$ (fold change) $<-1$, and the statistical significance ( $\mathrm{p}$-adjust value $<0.05$ ) was determined using the DEGseq package [26]. Venn diagrams were drawn using an online tool (http://bioinformatics.psb.ugent.be/webtools/Venn). GO and KEGG enrichment analyses were then performed on the differentially expressed unigenes using Goatools [27] and KOBAS software [28], respectively. Benjamini-hochberg correction was applied for GO and KEGG enrichment and significance set to adjusted P-value $<0.05$. We used the MapMan tool [29] for a graphical overview of the biotic stress response by default parameters. We used the pfam domains PF01397 and PF03936 to assess terpene synthase (TPS) genes in the P. acerifolia transcriptome assembly using the HMMER package [30] with a threshold of 0.01 . Phylogenetic trees were constructed using the neighbor-joining method as implemented in MEGA6.06 [31] with 1000 bootstrap replicates.

\subsection{Metabolome Analysis}

The leaf samples treatment method is the same as for RNA-seq except for six biological replicates; leaf metabolites were extracted using a previously described method [32]. Briefly, leaf samples were ground to a fine powder in liquid nitrogen, after which they were homogenized in methanol/chloroform and cold water. The extracted metabolites were then derivatized with methoxyamine hydrochloride and N-methyl-N-(trimethylsilyl) trifluoroacetamide (Sigma-Aldrich, St. Louis Missouri, USA). The instrument used was a 4 D GC $\times$ GC-TOF-MS (LECO, San Jose, USA) with DB-5MS capillary column $(30 \mathrm{~m} \times 250 \mu \mathrm{m} \times 0.25 \mu \mathrm{m}$, J\&W Scientific, Folsom, CA, USA). One microliter per sample was injected using a splitless model. With helium as the carrier gas, the gas flow rate through the column was $1 \mathrm{~mL} / \mathrm{min}$. The following temperature program was used, $2 \mathrm{~min}$ at $60^{\circ} \mathrm{C}$, increase to $200^{\circ} \mathrm{C}$ with $5{ }^{\circ} \mathrm{C} / \mathrm{min}$, then increase to $300{ }^{\circ} \mathrm{C}$ with $10{ }^{\circ} \mathrm{C} \mathrm{min} / \mathrm{min}$, and held $5 \mathrm{~min}$. The temperature of injection, 
transfer line, and ion source was 250,280 , and $250^{\circ} \mathrm{C}$, respectively. Mass spectrometry used full scan mode with $\mathrm{m} / \mathrm{z}$ range from 35 to 600,3 -min solvent delay. Data processing and analysis used Chroma TOF 4.3X software (LECO Corporation, St. Joseph, MI, United States). Compounds were identified by searching against the LECO/Fiehn metabolite Mass spectral library. Concentrations of tested metabolite were corrected with an internal standard, arabinose. PCA and PLADA were analyzed using the web tool metaboanalyst (https://www.metaboanalyst.ca) [33]. The metabolite quantities were compared by ANOVA in IBM SPSS Statistics software 20 [34]. The metabolic and transcriptomic data were integrated with the KEGG pathway mapping. Heat maps were drawn with R.

Supplementary Materials: The following are available online at http://www.mdpi.com/1422-0067/20/14/3465/s1.

Author Contributions: Conceived and designed the experiments: F.L., C.L.; Performed the experiments: F.L., D.L.; Analyzed the data: F.L., C.W.; Contributed reagents/materials/analysis tools: C.Q. wrote the paper: F.L., C.L., Y.D.

Acknowledgments: This work was funded by the Beijing Outstanding Talents Cultivation Youth Backbone Project (2017000020060G116) and the Project of Regional collaborative innovation of Beijing Academy of Agriculture and Forestry Sciences (grant No. KJCX20170709).

Conflicts of Interest: The authors declare no conflict of interest.

\section{References}

1. Halbert, S.E.; Meeker, J.R. The sycamore lace bug, Corythucha ciliata (Say) (Hemiptera: Tingidae). Ann. Entomol. Soc. Am. 1983, 76, 262-265.

2. Li, F.; Fu, N.; Zhang, L.; Jiao, M.; Peng, L.; Xu, Y.; Luo, C. Advances in biology, chemical ecology and control of the sycamore lace bug, Corythucha ciliata (Hemiptera: Tingidae). Acta Entomol. Sin. 2018, 61, 1076-1086.

3. Yu, L.; Liu, J.; Chen, Z.; Xu, S.; Ren, Z.; Wang, D.; Geng, K.; Zhang, Q.; Pei, W.; Kong, C. First Report of Stem die-back on Platanus $\times$ acerifolia Caused by Neofusicoccum parvum in China. Plant Dis. 2016. [CrossRef]

4. Ju, R.; Li, B.; Ma, K. Sycamore lace bug, Corythucha ciliata, an invasive alien pest rapidly spreading in urban China. Biodivers. Sci. 2010, 18, 638-646.

5. Li, F.; Fu, N.; Li, D.; Chang, H.; Qu, C.; Wang, R.; Xu, Y.; Luo, C. Identification of an Alarm Pheromone-Binding Chemosensory Protein From the Invasive Sycamore Lace Bug Corythucha ciliata (Say). Front. Physiol. 2018, 9, 354. [CrossRef] [PubMed]

6. Li, F.; Yang, S.; Fu, N.; Qu, C.; Liu, J.; Wang, R.; Xu, Y.; Luo, C. Volatile profiles of Platanus acerifolia leaves and their behavioral effects on Corythucha ciliata (Hemiptera: Tingidae). Chin. J. Appl. Entomol. 2017, 54, $207-213$.

7. Li, F.; Li, W.; Lin, Y.; Pickett, J.A.; Birkett, M.A.; Wu, K.; Wang, G.; Zhou, J.J. Expression of Lima Bean Terpene Synthases in Rice Enhances Recruitment of a Beneficial Enemy of a Major Rice Pest. Plant Cell Environ. 2018, 41, 111-120. [CrossRef]

8. Mierziak, J.; Kostyn, K.; Kulma, A. Flavonoids as important molecules of plant interactions with the environment. Molecules 2014, 19, 16240-16265. [CrossRef]

9. Chadwick, D.J.; Goode, J.A. Insect-plant interactions and induced plant defence; John Wiley \& Sons: Hoboken, NJ, USA, 2008; p. 223.

10. Li, F.; Fu, N.; Qu, C.; Wang, R.; Xu, Y.; Luo, C. Understanding the mechanisms of dormancy in an invasive alien Sycamore lace bug, Corythucha ciliata through transcript and metabolite profiling. Sci. Rep. 2017, 7, 2631. [CrossRef]

11. Chen, S.; Yanqing, Z.; Yaru, C.; Jia, G. fastp: An ultra-fast all-in-one FASTQ preprocessor. Bioinformatics 2018, 34, 884-890. [CrossRef]

12. Grabherr, M.G.; Haas, B.J.; Yassour, M.; Levin, J.Z.; Thompson, D.A.; Amit, I.; Xian, A.; Lin, F.; Raychowdhury, R.; Zeng, Q. Trinity: Reconstructing a full-length transcriptome without a genome from RNA-Seq data. Nat. Biotechnol. 2011, 29, 644-652. [CrossRef] [PubMed]

13. Li, B.; Dewey, C.N. RSEM: Accurate transcript quantification from RNA-Seq data with or without a reference genome. BMC Bioinform. 2011, 12, 323. [CrossRef] [PubMed]

14. Wang, L.; Feng, Z.; Wang, X.; Wang, X.; Zhang, X. DEGseq: An R package for identifying differentially expressed genes from RNA-seq data. Bioinformatics 2010, 26, 136-138. [CrossRef] [PubMed] 
15. Klopfenstein, D.V.; Zhang, L.; Pedersen, B.S.; Ramírez, F.; Vesztrocy, A.W.; Naldi, A.; Mungall, C.J.; Yunes, J.M.; Botvinnik, O.; Weigel, M. GOATOOLS: A Python library for Gene Ontology analyses. Sci. Rep. 2018, 8, 10872. [CrossRef] [PubMed]

16. Wu, J.; Mao, X.; Cai, T.; Luo, J.; Wei, L. KOBAS server: A web-based platform for automated annotation and pathway identification. Nucleic Acids Res. 2006, 34, W720-W724. [CrossRef] [PubMed]

17. Usadel, B.; Poree, F.; Nagel, A.; Lohse, M.; Czedik-Eysenberg, A.; Stitt, M. A guide to using MapMan to visualize and compare Omics data in plants: A case study in the crop species, Maize. Plant Cell Environ. 2010, 32, 1211-1229. [CrossRef] [PubMed]

18. Wistrand, M.; Sonnhammer, E.L. Improved profile HMM performance by assessment of critical algorithmic features in SAM and HMMER. BMC Bioinform. 2005, 6, 99. [CrossRef] [PubMed]

19. Tamura, K.; Stecher, G.; Peterson, D.; Filipski, A.; Kumar, S. MEGA6: Molecular Evolutionary Genetics Analysis Version 6.0. Mol. Biol. Evol. 2013, 30, 2725-2729. [CrossRef]

20. Jan, L.; Nicolas, S.; Joachim, K.; Lothar, W.; Fernie, A.R. Gas chromatography mass spectrometry-based metabolite profiling in plants. Nat. Protoc. 2006, 1, 387-396.

21. Xia, J.; Psychogios, N.; Young, N.; Wishart, D.S. MetaboAnalyst: A web server for metabolomic data analysis and interpretation. Nucleic Acids Res. 2009, 37, W652-W660. [CrossRef]

22. George, D. IBM SPSS Statistics 21 Step by Step: A Simple Guide and Reference; Pearson Higher Education: London, UK, 2013.

23. Zhou, G.; Wang, X.; Yan, F.; Wang, X.; Li, R.; Cheng, J.; Lou, Y. Genome-wide transcriptional changes and defence-related chemical profiling of rice in response to infestation by the rice striped stem borer Chilo Suppressalis. Physiol. Plant. 2011, 143, 21-40. [CrossRef] [PubMed]

24. De, V.M.; Van Oosten, V.R.; Van Poecke, R.M.; Van Pelt, J.A.; Pozo, M.J.; Mueller, M.J.; Buchala, A.J.; Métraux, J.P.; Van Loon, L.C.; Dicke, M. Signal signature and transcriptome changes of Arabidopsis during pathogen and insect attack. Mol. Plant-Microbe Interact. 2005, 18, 923.

25. Ehlting, J.; Chowrira, S.G.; Mattheus, N.; Aeschliman, D.S.; Arimura, G.I.; Bohlmann, J. Comparative transcriptome analysis of Arabidopsis thaliana infested by diamond back moth (Plutella xylostella) larvae reveals signatures of stress response, secondary metabolism, and signalling. BMC Genom. 2008, 9, 154. [CrossRef] [PubMed]

26. Lortzing, T.; Steppuhn, A. Jasmonate signalling in plants shapes plant-insect interaction ecology. Curr. Opin. Insect Sci. 2016, 14, 32-39. [CrossRef] [PubMed]

27. Fu, N.; Jia, L.; Cheng, Q.; Ran, W.; Xu, Y.; Chen, L.; Li, F. Analysis of Corythucha ciliata CcilCSP1 Structure and Prediction of Its Binding to Host-Plant Volatiles. Scientia Silvae Sinicae 2017, 10, 109-117.

28. Wink, M. Plant Secondary Metabolites Modulate Insect Behavior-Steps Toward Addiction? Front. Physiol. 2018, 9, 364. [CrossRef]

29. Gols, R. Direct and indirect chemical defences against insects in a multitrophic framework. Plant Cell Environ. 2014, 37, 1741-1752. [CrossRef] [PubMed]

30. Simmonds, M.S.J. Flavonoid-insect interactions: Recent advances in our knowledge. Phytochemistry 2003, 64, 21-30. [CrossRef]

31. Simmonds, M.S.J. Importance of flavonoids in insect-plant interactions: Feeding and oviposition. Phytochemistry 2001, 56, 245-252. [CrossRef]

32. Punyasiri, P.A.N.; Abeysinghe, I.S.B.; Kumar, V. Preformed and Induced Chemical Resistance of Tea Leaf Against Exobasidium vexans Infection. J. Chem. Ecol. 2005, 31, 1315-1324. [CrossRef]

33. Vandoorn, A.; Vos, M.D. Resistance to sap-sucking insects in modern-day agriculture. Front. Plant Sci. 2013, 4, 222. [CrossRef] [PubMed]

34. Namo Dubey, K.S. Role of NBS-LRR Proteins in Plant Defense. Mol. Asp. Plant-Pathog. Interact. 2018, 115-138. [CrossRef]

(C) 2019 by the authors. Licensee MDPI, Basel, Switzerland. This article is an open access article distributed under the terms and conditions of the Creative Commons Attribution (CC BY) license (http://creativecommons.org/licenses/by/4.0/). 